INFLAMMATORY BOWEL DISEASE

\title{
CD40 antisense oligonucleotide inhibition of trinitrobenzene sulphonic acid induced rat colitis
}

\author{
D Gao, A H Wagner, S Fankhaenel, T Stojanovic, S Schweyer, S Panzner, M Hecker
}

Gut 2005;54:70-77. doi: 10.1136/gut.2003.029587

See end of article for authors' affiliations

.....................

Correspondence to: Professor $M$ Hecker, Institut fur Physiologie und Pathophysiologie, Ruprecht-Karls-Universität Heidelberg, Im

Nevenheimer Feld 326 D-69120, Heidelberg, Germany; hecker@ physiologie.uni.hd.de

Revised version received 17 May 2004

Accepted for publication 3 June 2004
Background: CD154/CD40 interactions play a pivotal role both in humoral and cellular immune responses. Their involvement in the pathogenesis of chronic inflammatory bowel disease (IBD) has been revealed by increased expression of CD40 and CD154 in the inflamed mucosa of patients and the therapeutic effects of anti-CD154 antibodies in experimental colitis. Because of adverse side effects however, the use of such antibodies in patients with IBD may be limited.

Aims: An alternative approach to blocking CD154/CD40 interactions by employing a CD40 antisense oligonucleotide (ODN) was explored.

Results: After sequencing of the rat CD40 gene, five antisense ODNs were designed, of which one (rAS3) effectively downregulated CD40 expression in rat vascular smooth muscle cells as well as the subsequent changes in gene expression in response to CD40 stimulation. The therapeutic potency of rAS3 was evaluated in the 2,4,6-trinitrobenzene sulphonic acid (TNBS) induced colitis model of the rat. Single intracolonic injection of a liposomal formulation of rAS3 either prior to or post colitis induction markedly suppressed the inflammatory reaction in these animals monitored both macroscopically and microscopically over one week, while application of a scrambled control ODN had no such effects. Moreover, reverse transcription-polymerase chain reaction analyses revealed reduced expression of vascular cell adhesion molecule 1, interleukin 12 p40, and monocyte chemoatractive protein 1 in the inflamed mucosa, which in turn may have contributed to the decrease in leucocyte infiltration judged by immunohistochemistry.

Conclusions: These results suggest that CD40 antisense ODNs effectively interfere with CD154/CD40 interactions in vivo and, therefore, may provide a novel approach to the treatment of patients with chronic IBD.
C D40 is a cell surface receptor that belongs to the tumour necrosis factor receptor family. It is constitutively expressed by B cells, monocytes/macrophages, and dendritic cells. In addition to classic antigen presenting cells (APCs), CD40 expression is also found in endothelial cells, fibroblasts, and smooth muscle cells. Its corresponding ligand, CD154, is mainly expressed on activated $\mathrm{CD}^{+} \mathrm{T}$ helper cells. ${ }^{12}$

The pivotal role of $\mathrm{CD154/CD40}$ interactions in the regulation of immune responses has been demonstrated by the severe immunodeficiency caused through deletion or mutation of either the CD40 or CD154 gene. ${ }^{3-5}$ Moreover, activation of CD40 in dendritic cells and macrophages appears to augment their antigen presenting functions through upregulation of expression of costimulatory molecules on their surface and release of proinflammatory cytokines. ${ }^{12}$ CD40 engagement also enhances expression of adhesion molecules (intracellular adhesion molecule (ICAM-1), vascular cell adhesion molecule 1 (VCAM-1), and E-selectin) on the surface of endothelial cells which in turn facilitates the recruitment and activation of leucocytes at the site of inflammation. ${ }^{67}$ These findings suggest that CD154/ CD40 interactions play an important role in the pathogenesis of chronic inflammatory diseases.

Inflammatory bowel disease (IBD), mainly referring to Crohn's disease and ulcerative colitis, is characterised by chronic inflammation of the gastrointestinal tract. Although their aetiology remains unknown, inappropriate and sustained activation of the mucosal immune system seems crucial to the development of these diseases. There is increasing evidence that CD154/CD40 costimulation plays a role in the pathogenesis of IBD. CD154 transgenic mice spontaneously develop lethal IBD, characterised by infiltration of CD154 positive T cells and CD40 positive monocytes into the diseased tissue. ${ }^{8}$ In patients with Crohn's disease, an increased number of CD154 positive T cells has been detected in the lamina propria together with several CD40 positive cells, ${ }^{9}{ }^{10}$ the majority of which were identified as B cells or macrophages. Interestingly, endothelial cells also stained positive for CD40. This finding is in line with the view that the vascular endothelium plays an important role in the maintenance of intestinal integrity, and that blockade of CD154/CD40 interactions may exert a potent therapeutic effect in Crohn's disease.

However, administration of anti-CD154 antibodies, although clearly effective in a variety of animal models of inflammation, including colitis, revealed major thromboembolic complications both in experimental animals ${ }^{11}$ and in patients. ${ }^{12}$ One likely explanation for this adverse effect is that soluble CD154 plays an important role in thrombus stabilisation. ${ }^{13}$ Anti-CD40 antibodies, on the other hand, tend to activate CD40 expressing cells, ${ }^{14}$ and low molecular weight antagonists for CD40 are not yet available. We hypothesised therefore that targeting CD40 expression in APCs, including endothelial cells, may provide a feasible therapeutic alternative. To this end, we have designed various CD40 antisense

Abbreviations: APC, antigen presenting cells; EF-2, elongation factor 2 ; IBD, inflammatory bowel disease; ODN, oligonucleotide; RACE, rapid amplification of cDNA ends; TNBS, 2,4,6-trinitrobenzene sulphonic acid; VSMC, vascular smooth muscle cells; ICAM-1, intercellular adhesion molecule 1; VCAM-1, vascular cell adhesion molecule 1; RT-PCR, reverse transcription-polymerase chain reaction; IL-12, interleukin 12; MCP-1, monocyte chemoatractive protein 1; IFN- $\gamma$, interferon $\gamma$; TNF- $\alpha$, tumour necrosis factor $\alpha$ 
oligonucleotides (ODNs) and studied their therapeutic effects in a rat model of Crohn's disease.

\section{MATERIALS AND METHODS}

\section{Elucidation of the sequence of rat CD40 mRNA}

Starting from the partial sequence of rat CD40 mRNA (GenBank No AF241231), the rapid amplification of cDNA ends (RACE) method was employed to elucidate the coding sequence of its 5'-end using the 5'-RACE kit from Life Technologies (Karlsruhe, Germany). Rat CD40 gene specific primers used for these experiments were: rGSP1, $5^{\prime}$-ATC CTC ACA GCT TGT CCA-3'; rGSP2, 5'-ACA TGC CTC GCA ATC CTT GC-3'; and rGSP3, 5'-GAG GCA AAG ACA CCA T-3'. The amplified rat CD40 fragment was separated on a $1 \%$ agarose gel, purified using the QIAEX II kit (Qiagen, Hilden, Germany), and sequenced using a model 373 stretch DNA sequencer (Applied Biosystems, Weiterstadt, Germany).

\section{Cell culture}

Rat vascular smooth muscle cells (VSMCs) were obtained from rat aorta by the explant technique, as described previously, ${ }^{15}$ and cultured in Waymouth medium (Life Technologies) supplemented with $10 \%$ fetal bovine serum, $50 \mathrm{U} / \mathrm{ml}$ penicillin, $50 \mu \mathrm{g} / \mathrm{ml}$ streptomycin, and $1 \mu \mathrm{g} / \mathrm{ml}$ heparin until confluence (typically one week). For the experiments described herein, passage 3 cells derived from different animals were employed throughout.

The mouse myloma cell line P3xTB.A7 stably expressing human CD154 and the non-transfected P3x63Ag8.653 control cell line were cultured in RPMI 1640 medium (Life Technologies) with 10\% fetal bovine serum and antibiotics, as described previously. ${ }^{16}$

\section{Transfection of antisense ODNs in vitro}

CD40 antisense ODNs were designed as described in the results section below and synthesised by Eurogentec (Köln, Germany). Rat VSMCs were seeded onto six well multiwell plates, attaining approximately $90 \%$ confluence at the time of transfection. The transfection solution was prepared by mixing CD40 antisense or scrambled control ODNs (for example, $1 \mu \mathrm{M})$ and Lipofectin reagent $(2 \mu \mathrm{g} / \mathrm{ml}$; Life Technologies) according to the manufacturer's instructions. Cells were incubated with the transfection medium for four hours in the incubator before changing back to normal medium.

\section{Western blot analysis}

Western blot analysis of CD40 expression in rat VSMCs and rat colon samples was performed according to standard protocols. $^{15}$ A polyclonal rabbit antirat CD40 antibody (Biovision, San Diego, California, USA) was used as the primary antibody at a 1:500 dilution. A horseradish peroxidase conjugated goat antirabbit IgG antibody (SigmaAldrich, Taufkirchen, Germany; 1:3000 dilution) was used as the secondary antibody. Equal loading and transfer of protein was verified by reprobing the membrane with a monoclonal anti- $\beta$-actin antibody (Sigma-Aldrich; 1:3000 dilution). The intensity of the immunoreactive bands was quantified by densitometry. ${ }^{15}$

\section{Reverse transcription-polymerase chain reaction (RT-PCR) analysis}

Total RNA was isolated from cultured rat VSMCs or rat colon specimens using the RNeasy Mini kit (Qiagen). Semi quantitative RT-PCR analyses for rat CD154, VCAM-1, interleukin 12 (IL-12) p40, monocyte chemoatractive protein 1 (MCP-1), and elongation factor-2 (EF-2) as an internal standard were performed as described previously. ${ }^{16}$ Primers for the amplification of interferon $\gamma($ IFN- $\gamma)$ cDNA were
5'-CAT GGA TGC TAT GGA AGG AAA GAG C-3' (forward) and 5'-CTT ATG TTG TTG CTG ATG GCC TGG-3' (reverse). The identity of all amplification products was verified by cloning and sequencing.

\section{Animals}

Non-fasted male Wistar rats (Winkelmann, Borchen, Germany) weighting 200-250 g were used for the experiments. All animals were fed standard rat chow and had free access to water and food. They were kept according to German legislation on the protection of animals, with a 12 hour day and night cycle.

\section{Colitis induction}

Colitis was induced using a single intracolonic application of 2,4,6-trinitrobenzene sulphonic acid (TNBS), as described previously. ${ }^{16}$ The inducing mixture was prepared by dissolving $20 \mathrm{mg}$ of TNBS (Sigma-Aldrich) in 35\% ethanol with $0.9 \% \mathrm{NaCl}$ in a total volume of $136 \mu \mathrm{l}$. Rats were placed under light ether anaesthesia. The mixture was administered with an $8 \mathrm{~cm}$ long catheter inserted through the anal canal into the descending colon. Expelling of the total volume was ensured by additional air injection. After removing the catheter, rats were held in a headfirst position for 30 seconds to avoid flowing out of the enema. Rats were kept under normal condition after the intervention.

\section{Transfection of antisense ODNs in vivo}

For application of the antisense and scrambled control ODNs, they were encapsulated in liposomes. In brief, $14.3 \mu \mathrm{mol}$ of charge reversible lipid Na-(L-histidinyl)-cholesterol- hemisuccinate, $14.3 \mu \mathrm{mol}$ of cholesterol, and $42.9 \mu \mathrm{mol}$ of 1-palmitoyl-2 oleoyl-sn-glycero- 3-phosphocholine were dissolved in chloroform in a $10 \mathrm{ml}$ round bottomed flask. After evaporation of the solvent, the lipid film was further dried under vacuum overnight. Buffer $(1 \mathrm{ml})$ containing $10 \mathrm{mM}$ sodium acetate, $150 \mathrm{mM}$ sodium chloride, and $1 \mathrm{mg} / \mathrm{ml}$ of the ODN, pH 4.5, was used for hydration. The initial suspension was sonicated for five minutes and frozen $\left(-70^{\circ} \mathrm{C}\right)$. After thawing, liposomes were extruded 15 times through polycarbonate filters having a pore diameter of $400 \mathrm{~nm}$. Liposomes were adjusted to $\mathrm{pH} 7.5$ using $\mathrm{l} \mathrm{M}$

\begin{tabular}{ll}
$\begin{array}{l}\text { Table } 1 \text { Criteria for microscopic scoring of colonic } \\
\text { damage }\end{array}$ & \\
\hline Parameter & Score \\
\hline Ulceration & \\
No & 0 \\
Minor & 1 \\
Major & 2 \\
Inflammation & 0 \\
None & 1 \\
Minor & 2 \\
Major & 3 \\
Severe & \\
Depth of lesion & 0 \\
None & 1 \\
Superficial & 2 \\
One third & 3 \\
Two thirds & 4 \\
Transmural & \\
Fibrosis & 0 \\
None & 1 \\
Minor & 2 \\
Major & \\
Lymphocyte infiltration & 0 \\
No & 1 \\
Yes & $0-12$ \\
Total score & \\
\hline & \\
\hline & \\
\hline
\end{tabular}


HEPES buffer, stored at $4{ }^{\circ} \mathrm{C}$, and readjusted to $\mathrm{pH} 4.5$ using $1 \mathrm{M}$ sodium acetate immediately before use. They were administered by intracolonic injection $(2.7 \mu \mathrm{g}$ DNA in a total volume of $100 \mu \mathrm{l}$ ) four hours prior to and/or three days post colitis induction.

\section{Assessment of colonic inflammation and damage}

Seven days after induction of colitis, animals were sacrificed under deep ether anaesthesia. The colon was removed and opened longitudinally. Colonic inflammation and damage were assessed macroscopically, as described previously. ${ }^{16}$ Samples from both the affected descending colon and from the unaffected ascending colon were collected for histological and RT-PCR analysis. The blocking effect of the antisense ODN on CD40 expression in vivo was evaluated on day 2 post colitis induction by immunohistochemistry and western blot analysis.

\section{(Immuno)histochemistry}

Colon samples were fixed in phosphate buffered saline containing $4 \%$ formaldehyde. Paraffin embedded sections $(5 \mu \mathrm{m})$ were stained with haematoxylin/eosin followed by light microscopic evaluation of tissue injury, according to the criteria described in table 1 , by two independent investigators in a blinded fashion.

For immunohistochemical staining, serial sections were incubated for one hour with blocking buffer (10\% bovine serum albumin in phosphate buffered saline) followed by overnight incubation at $4^{\circ} \mathrm{C}$ with a mouse antirat ED-1 antibody (1:750 dilution; Dako, Cytomation, Hamburg, Germany) or a mouse antirat CD3 antibody (1:75 dilution; Dako), respectively. Monocytes and T cells were visualised using the avidin-biotin-peroxidase complex technique with biotinylated mouse antigoat/sheep IgG as secondary antibody, peroxidase conjugated avidin as a label, and 3-amino9-ethylcarbazole as a chromogen. Granulocytes were detected using the Naphtol AS-D chloroacetate esterase kit (SigmaAldrich) according to the manufacturer's instructions. Dewaxed sections were incubated in the staining solution for 15 minutes at $37^{\circ} \mathrm{C}$ in the dark and counterstained with haematoxylin.

Positively stained cells were counted using the Metamorph version 6.01 software package (Universal Imaging Corp, Downingtown, Pennsylvania, USA) in a blinded fashion.

For detection of CD40, fresh colon samples were embedded in Tissue-Tek (Sakura Finetek, Zoeterwoude, the Netherlands) and frozen immediately in liquid nitrogen chilled isopentane. Frozen sections $(10 \mu \mathrm{m})$ were blocked with goat serum and incubated with the rabbit antirat CD40 antibody (1:100 dilution; Biovision) as the primary antibody and a biotinylated goat antirabbit IgG antibody (1:500 dilution; Dako) as the secondary antibody. Positive immunoreactivity was visualised using the avidin-biotin-peroxidase complex technique, as described above.

Table 2 Antisense oligonucleotides (ODNs) against rat CD40 (including the scrambled control oligonucleotide)

\begin{tabular}{lll}
\hline ODN & Sequence & Location \\
\hline rAS1 & $A^{*} A^{*} G^{*} G C A G C A T T G C A^{*} G^{*} A^{*}$ & -6 to +10 \\
rAS2 & $A^{*} C^{*} C^{*} G C T G T C A A C A A G C A^{*} G^{*} C^{*}$ & +35 to +53 \\
rAS3 & $T^{*} C^{*} C^{*}$ TAGATGGACCGCT*G*T* & +46 to +63 \\
rAS4 & $T^{*} A^{*} A^{*}$ CACACTGTCCT $A^{*} G^{*}$ & +58 to +73 \\
rAS5 & $T^{*} C^{*} T^{*} C$ CCTGTCCAG*T $T^{*}$ & +208 to +223 \\
rScr & $C^{*} T^{*} C^{*}$ TCACGTCGAGGTG* $T^{*} A^{*}$ & Srambled ODN \\
\hline *Phosphorothioate modified nucleotide. & \\
\hline
\end{tabular}
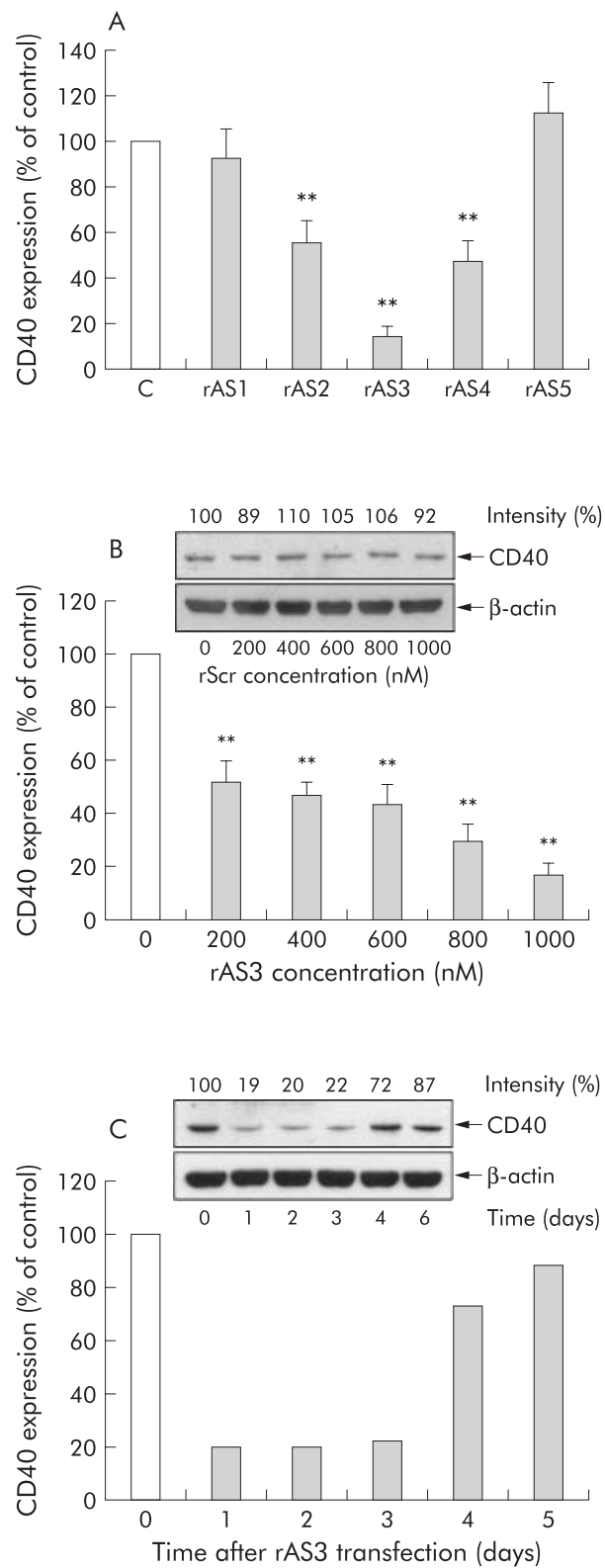

Figure 1 Effects of the antisense oligonucleotides (ODNs) on CD40 protein abundance. Cultured rat vascular smooth muscle cells were transfected with different antisense ODNs at a concentration of $1 \mu \mathrm{M}$ (A). Cells were harvested 24 hours after transfection for western blot analysis. The inhibitory effect of rAS3 on CD40 protein was further characterised at different concentrations (B) and at different time intervals post transfection (C). For comparison, the effect of the scrambled control ODN (rScr) on CD40 expression is also shown (B insert). CD40 expression was estimated by densitometry analysis and expressed as relative intensity (\%) compared with untreated control cells. Statistical summary of the results of three $(A, B)$ and two individual experiments $(C)$ are shown, with a representative western blot using $\beta$-actin as an internal standard. ${ }^{* *} \mathrm{p}<0.01$ versus untreated control.

\section{Statistical analysis}

Unless indicated otherwise, all data in the figures and text are expressed as means (SD) of $\mathrm{n}$ independent observations. Statistical evaluation was performed by one way analysis of variance followed by a Bonferroni multiple comparisons test (comparison of three or more groups) or unpaired two tailed Student's $t$ test (comparison of two groups) with the Instat for Windows version 3.05 software package (GraphPad Software, San Diego, California, USA). A p value $<0.05$ was considered statistically significant. 


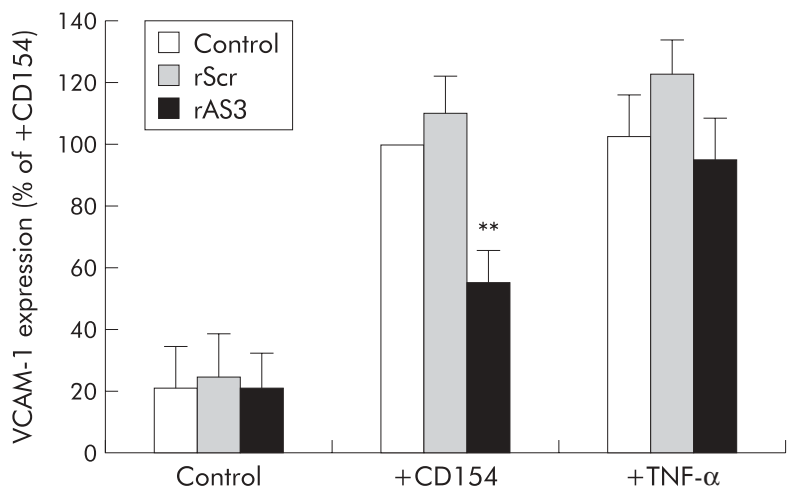

Figure 2 Blocking effect of rAS3 on CD40 dependent vascular cell adhesion molecule 1 (VCAM-1) expression in rat vascular smooth muscle cells. Cells were transfected with rAS3 or rScr $(1 \mu \mathrm{M})$. After 24 hours, they were stimulated with tumour necrosis factor $\alpha$ (TNF- $\alpha$ $1000 \mathrm{U} / \mathrm{ml}$ ) or by coculturing with CD154 positive P3xTB.A7 cells $\left(2 \times 10^{6} \mathrm{cell} / \mathrm{m} / \mathrm{m}\right)$ for 12 hours. VCAM-1 mRNA abundance was measured by semi quantitative reverse transcription-polymerase chain reaction, using elongation factor 2 as an internal standard. Statistical summary of VCAM-1 mRNA abundance is expressed as percentage of the CD154 stimulated control. $n=3-5 ;{ }^{* *} p<0.01$ versus control in the same group.

\section{RESULTS}

\section{Design of rat CD40 antisense ODNs}

Starting from the partial sequence, ${ }^{15} 5^{\prime}$-RACE was successfully employed to elucidate the $5^{\prime}$-end of rat CD40 mRNA, including the transcription start site (GenBank No AF241231). Thereafter, five antisense ODNs against rat CD40 mRNA were designed corresponding to different locations in this region (table 2). To avoid degradation by exonucleases, ODNs were protected by incorporating nuclease resistant phosphorothioate bonds in the last three nucleotides at the $5^{\prime}$ - and $3^{\prime}$-ends, respectively.

\section{Antisense ODN downregulation of CD40 protein}

We have previously shown that CD40 is expressed by rat VSMCs. ${ }^{15}$ Initially, the antisense ODNs were transfected into these cells at a concentration of $1 \mu \mathrm{M}$ and their effects determined at the protein level. As expected, the efficacy of the different antisense ODNs varied under these conditions (fig 1A) with rAS3 revealing the most pronounced inhibitory effect $(>80 \%$ decrease in CD40 protein abundance). Subsequent transfection of the cells with different concentrations of rAS3 revealed a significant effect on CD40 expression at only $0.2 \mu \mathrm{M}$ and a near maximum effect at
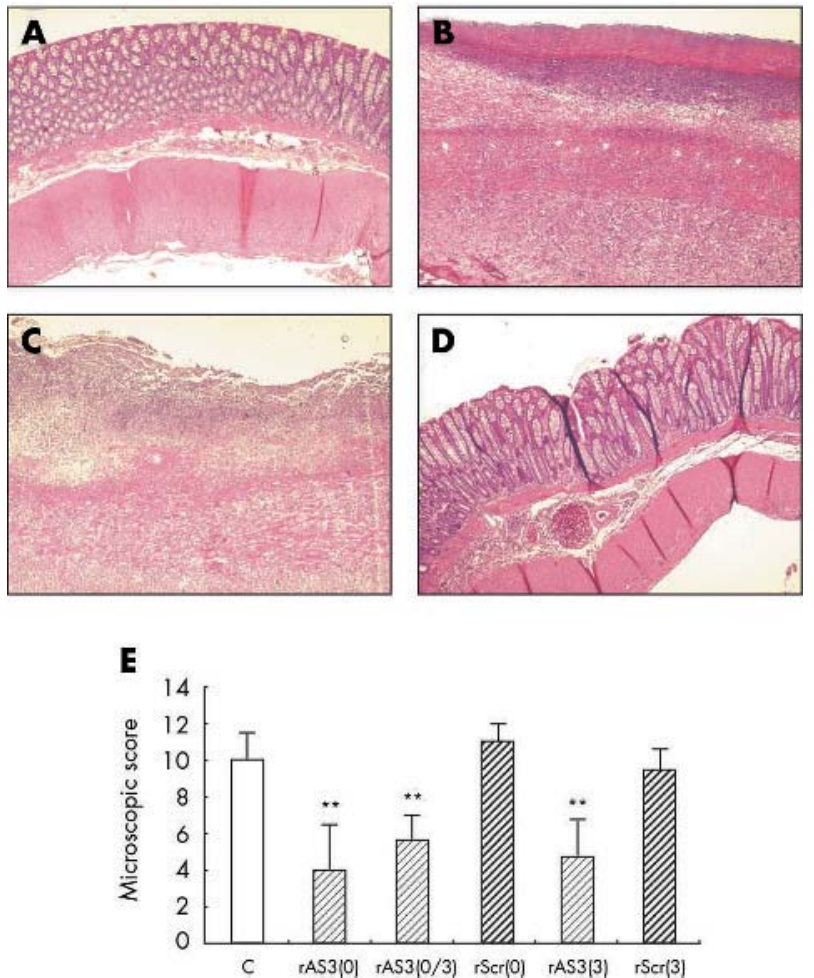

Figure 3 Histological analysis of CD40 antisense oligonucleotide (ODN) effects in 2,4,6-trinitrobenzene sulphonic acid (TNBS) induced colitis. Normal structure of the bowel wall in the unaffected ascending colon (A) but major changes in the affected descending colon of control animals (B) were seen, revealed by necrosis and thickening of the bowel wall as well as a prominent leucocyte infiltrate. Treatment prior to colitis induction with $r A S 3(D)$, but not $r S c r(C)$, resulted in significant protection against colitis development (haematoxylin/eosin staining, original $\times 50$ ). (E) Statistical summary comparing the histological analysis $(n=6-8$, ${ }^{* *} \mathrm{p}<0.01$ ) for the different treatment groups (rAS3, CD40 antisense ODN; rScr, scrambled control ODN; 0 , treated at four hours prior to colitis induction on day 0 ; and 3 , treated on the third day post colitis induction).

$1 \mu \mathrm{M}$ (fig $1 \mathrm{~B}$ ). In contrast, no inhibitory effect was detected with even the highest concentration of the scrambled control ODN (fig 1B insert). Moreover, to study the stability of the blocking effect, CD40 expression after rAS3 transfection was monitored for up to six days, demonstrating that downregulation of $\mathrm{CD} 40$ protein was maintained for three days (fig lC).

Table 3 Effects of liposome encapsulated oligonucleotides (ODNs) on macroscopic appearance in the colon of trinitrobenzene sulphonic acid treated animals

\begin{tabular}{lclllll}
\hline Parameter (range) & Control & rAS3(0) & rAS3(0/3) & rScr(0) & rAS3(3) & rScr(3) \\
\hline Diarrhoea (0-2) & $0.1(0.4)$ & $0.0(0.0)$ & $0.0(0.0)$ & $0.4(0.5)$ & $0.2(0.4)$ & $0.3(0.5)$ \\
General condition (0-1) & $0.3(0.3)$ & $0.0(0.0)$ & $0.0(0.0)$ & $0.0(0.0)$ & $0.0(0.0)$ & $0.3(0.5)$ \\
Adhesions (0-3) & $2.1(0.6)$ & $1.0(0.5)^{* *}$ & $1.3(1.0)$ & $2.1(0.7)$ & $1.3(1.0)$ & $2.0(0.6)$ \\
Perforation (0-1) & $0.3(0.5)$ & $0.0(0.0)$ & $0.3(0.5)$ & $0.4(0.5)$ & $0.2(0.4)^{* *}$ & $0.8(0.4)$ \\
Necrosis (0-4) & $2.9(0.6)$ & $1.3(1.3)^{* *}$ & $1.8(0.8)^{* *}$ & $3.4(0.5)$ & $1.8(0.8)$ & $2.7(0.8)$ \\
Ulceration (0-4) & $3.6(0.5)$ & $2.5(0.5)^{* *}$ & $2.3(0.5)^{* *}$ & $3.9(0.4)$ & $1.7(0.8)^{* *}$ & $3.7(0.8)$ \\
Oedema (0-1) & $1.0(0.0)$ & $1.0(0.0)$ & $0.8(0.4)$ & $1.0(0.0)$ & $0.8(0.4)$ & $1.0(0.0)$ \\
Megacolon (0-1) & $0.3(0.5)$ & $0.1(0.4)$ & $0.0(0.0)$ & $0.3(0.5)$ & $0.0(0.0)$ & $0.2(0.4)$ \\
Total score & $10.6(2.3)$ & $5.9(2.2)^{* *}$ & $6.7(2.6)^{* *}$ & $11.6(2.4)$ & $6.0(3.0)^{* *}$ & $11.0(2.9)$ \\
No of animals & 8 & 8 & 6 & 7 & 6 & 6 \\
\hline
\end{tabular}

Abbreviations indicate the different treatment regimes as follows: rAS3, CD40 antisense ODN; rScr, scrambled control ODN; 0 , treated at four hours prior to colitis induction on day $0 ; 3$, treated on the third day post colitis induction.

Values are mean (SD) score values rounded to one decimal.

${ }^{* *} p<0.01$ versus control. 

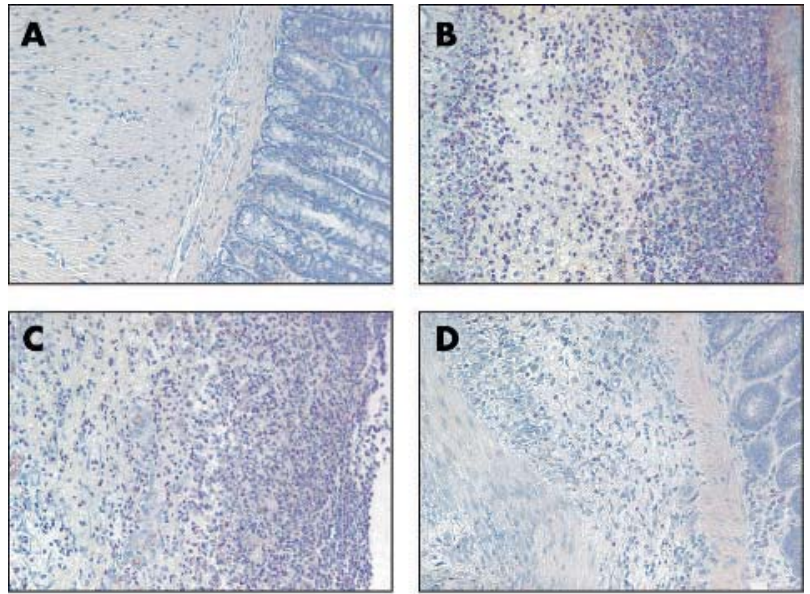

$\mathbf{E}$

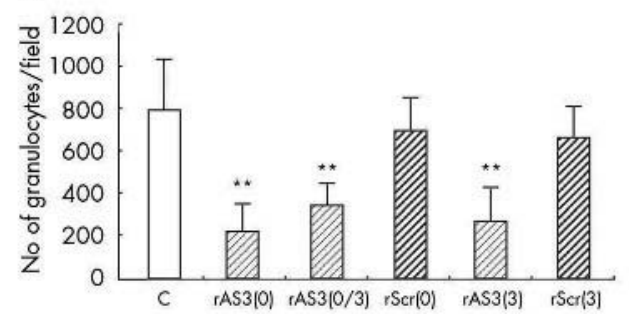

Figure 4 CD40 antisense oligonucleotide (ODN) effects on granulocyte infiltration in 2,4,6-trinitrobenzene sulphonic acid (TNBS) induced colitis. Sections were stained with Fast Red Violet LB (Sigma Diagnostics) for granulocytes in bright red colour. Only a few cells stained positive in the unaffected ascending colon (A) while a great number of granulocytes were observed in the descending colon of control animals (B). Treatment prior to colitis induction with rAS3 (D), but not $r S c r(C)$, resulted in a significant decrease in granulocyte count in the affected descending colon (original $\times 200)$. (E) Statistical analysis $\left(n=7-8,{ }^{* *} p<0.01\right)$

comparing the number of positive cells counted in each section for the different treatment groups (rAS3, CD40 antisense ODN; rScr, scrambled control ODN; 0 , treated at four hours prior to colitis induction on day 0 ; and 3 , treated on the third day post colitis induction).

\section{Attenuation of CD40 signalling in vitro}

Having established rAS3 as the most potent antisense ODN, we next assessed its ability to functionally block CD40 activation in cultured rat VSMCs. To this end, CD40 mediated VCAM-1 expression was used as a readout for CD40 stimulation. As shown in fig 2, VCAM-1 expression in rat VSMCs was markedly increased after stimulation with tumour necrosis factor $\alpha(\mathrm{TNF}-\alpha 1000 \mathrm{U} / \mathrm{ml})$ or CD154. In cells transfected with rAS3 but not the scrambled control ODN, CD154 induced VCAM-1 expression was significantly inhibited, while TNF- $\alpha$ stimulated VCAM-1 expression was not affected. These results demonstrate that rAS3 is a specific blocker of CD154/CD40 interactions, and encouraged us to investigate its potential therapeutic effects in vivo.

\section{CD40 antisense treatment prevents the development of severe colitis in vivo}

In male Wistar rats, single intracolonic injection of $20 \mathrm{mg}$ TNBS in 35\% ethanol reproducibly induces a transient Crohn's disease-like colitis with maximum inflammation at 3-5 days and spontaneous healing after approximately four weeks. ${ }^{16}$ After seven days, significant oedema formation together with focal ulcerations, necrosis, and adhesions was observed in the descending colon of the TNBS control group. With a single injection of rAS3 either four hours prior to or three days post colitis induction, the most severe signs of damage to the colon (that is, necrosis, ulceration, and
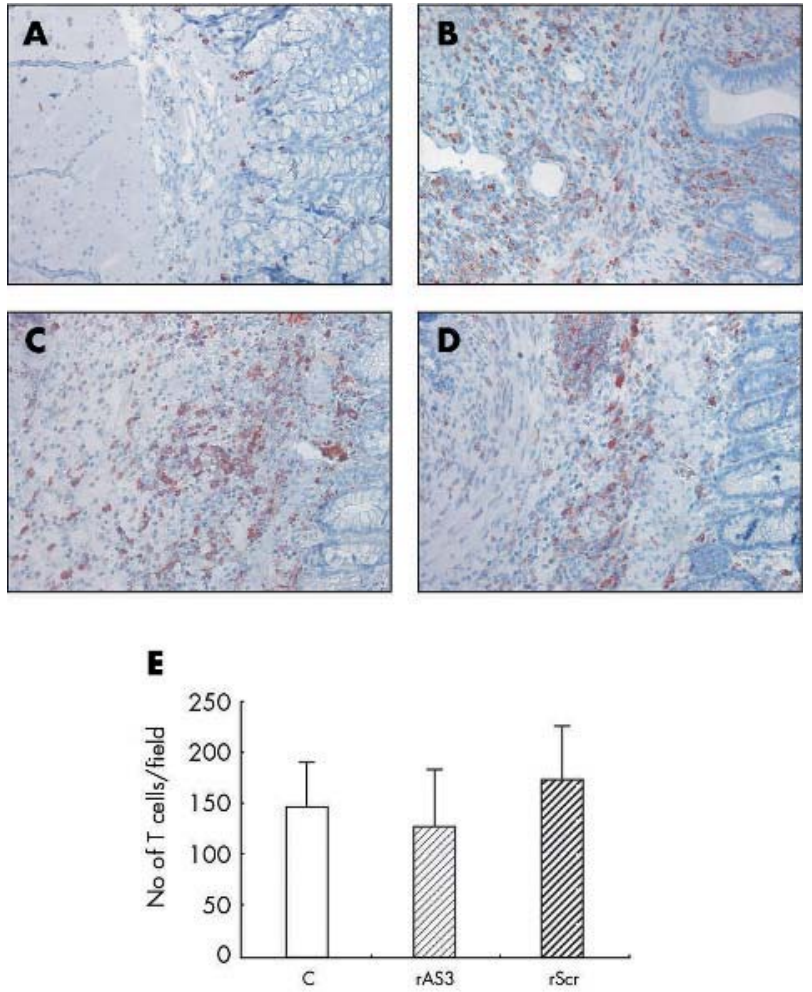

Figure $5 \mathrm{CD} 40$ antisense oligonucleotide effects on T cell infiltration in 2,4,6-trinitrobenzene sulphonic acid (TNBS) induced colitis. Sections were stained with the anti-CD3 antibody. Only a few cells stained positive in the unaffected ascending colon (A), while an appreciable number of $C D 3$ positive cells were observed in the descending colon of control (B), rScr treated (C), and rAS3 treated animals (D; original $\times 200)$. (E) Statistical analysis $(n=7-8)$ comparing the number of positive cells counted in each section for the control group, the rAS3 treated group, and the rScr treated group (four hours prior to colitis induction).

adhesions) were significantly reduced and, as a consequence, the overall macroscopic score (table 3). Repeat treatment with rAS3 at both time points did not improve the macroscopic score while the scrambled control ODN, irrespective of the time of application, failed to exert a therapeutic effect.

Histologically, damage to the bowel wall appeared to be discontinuous with areas of normal mucosa next to severely necrotic ones. In the descending colon of the TNBS control group, the mucosa was usually lost and the bowel wall thickened because of the prominent infiltration of leucocytes and moderate fibrosis (fig $3 \mathrm{~B}$ ). These changes in bowel wall structure were much less pronounced in rAS3 treated animals (fig 3D). Microscopic scoring also revealed that either pretreatment or delayed treatment with rAS3, but not with the scrambled control ODN, resulted in a significant improvement in colonic inflammation (fig 3E).

Histochemistry demonstrated prominent infiltration of granulocytes into the affected colon of both control and rScr treated rats, especially in the most severely damaged regions (fig 4B, C, respectively). In contrast, in colonic sections from rAS3 treated animals, only a few granulocytes were observed in the mucosa and submucosa propria (fig 4D). Immunohistochemistry revealed that CD3 (T cells) and ED-1 positive cells (monocytes) were mostly located in the less inflamed regions. Treatment with the antisense ODN failed to significantly reduce either the T cell (fig 5) or monocyte count (not shown). 

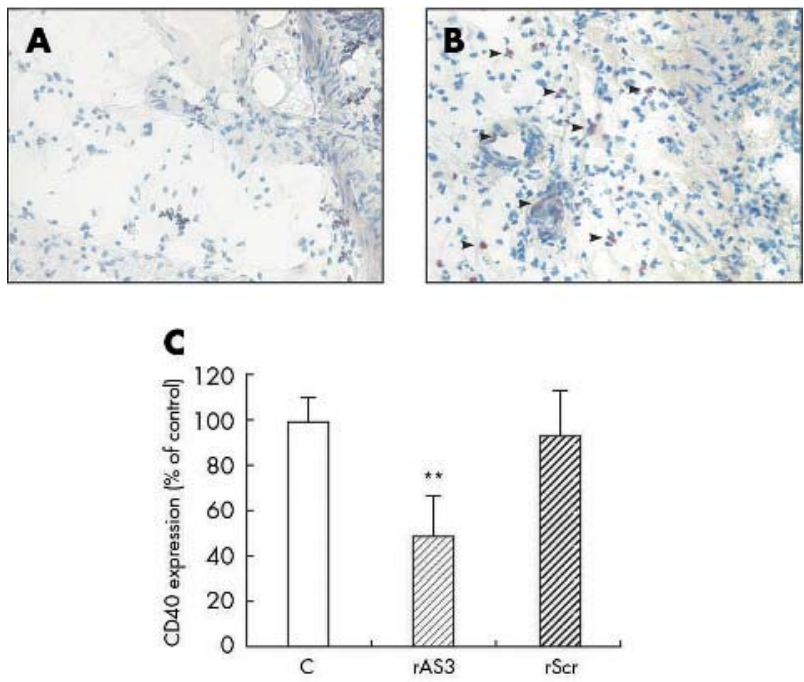

Figure 6 Blocking effect of rAS3 on CD40 expression in vivo. Animals were sacrificed on the second day after oligonucleotide administration and colitis induction. Colon sections were stained with the anti-CD40 antibody. In the unaffected ascending colon, only a few cells stained positive (A) while in the descending colon of 2,4,6-trinitrobenzene sulphonic acid (TNBS) treated controls an appreciable number of CD40 positive cells were detected ( $B$, indicated by arrows), which were identified as monocytes and endothelial cells. Western blot analysis (C) was employed to quantify the decrease in CD40 protein in the descending colon of rAS3 treated compared with control or rScr treated rats. $n=3 ;{ }^{* *} p<0.01$ versus untreated control.

Furthermore, CD40 expression in the unaffected ascending colon was hard to detect either by immunohistochemistry (fig 6A) or western blot analysis while in the inflamed descending colon an appreciable number of CD40 positive cells was verified (fig 6B). Most of these cells appeared to be infiltrated monocytes but some endothelial cells also stained positive. Treatment with rAS3 but not rScr clearly reduced CD40 expression in the inflamed colon, as quantified by western blot analysis (fig 6C).

Based on RT-PCR analysis, expression of CD154, VCAM-1, and IL-12 p40 was significantly increased in the descending colon of TNBS treated animals compared with the unaffected ascending colon (fig 7), indicative of the ongoing inflammation. In contrast, IFN- $\gamma$ mRNA abundance did not differ between the two sites (not shown). Antisense ODN treatment failed to affect the presumably $\mathrm{T}$ cell associated increase in CD154 expression (fig 7A) but significantly reduced that of VCAM-1 (fig 7B) and IL-12 p40 (fig 7C). MCP-1 mRNA expression was seemingly absent in the ascending colon but readily detectable in the descending colon of TNBS treated animals. Therefore, changes in MCP-1 mRNA abundance had to be expressed relative to the level in the descending colon of the TNBS control group, and this was strongly diminished in rats receiving the antisense ODN (mean 14 (SD 8)\% of TNBS control, $\mathrm{n}=8 ; \mathrm{p}<0.01$ ) but not the scrambled control ODN (106 (52)\%, $\mathrm{n}=7)$.

\section{DISCUSSION}

CD154/CD40 interactions play a pivotal role in both humoral and cellular immune responses and as such represent an attractive target for immune modulation in a number of diseases. To know the sequence of a target mRNA is the first requirement for antisense ODN design. Considering that alternative splicing occurs in the region between exons 5 and 9 of the human and mouse gene, ${ }^{17}$ we focused on the $5^{\prime}$-end of rat CD40 mRNA, the sequence of which was delineated by 5'-RACE.
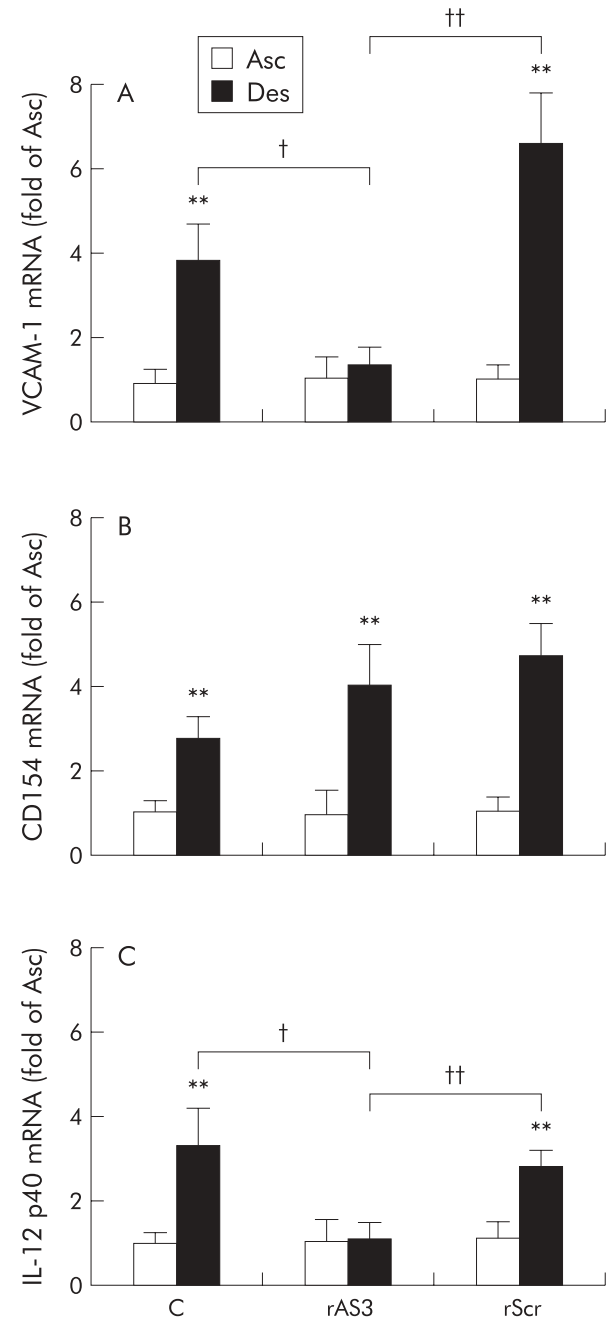

Figure 7 CD40 antisense oligonucleotide (rAS3) effects compared with control or $\mathrm{rScr}$ treatment, on vascular cell adhesion molecule 1 (VCAM-1) (A), CD154 (B), and interleukin 12 (IL-12) p40 (C) mRNA abundance in the colon of 2,4,6-trinitrobenzene sulphonic acid (TNBS) treated rats. Samples of both the ascending (Asc; that is, unaffected region) and descending (Des; that is, affected region) colon were collected seven days post colitis induction. mRNA abundance (relative to the mRNA level of the housekeeping gene elongation factor 2) for each gene product is statistically summarised ( $n=8,{ }^{* *} p<0.01$ versus Asc; $\mathrm{tp}<0.05,+\uparrow \mathrm{p}<0.01$ as indicated)

Antisense ODNs encompassing different regions of a mRNA molecule usually behave differently in blocking target protein expression, and this may depend on the three dimensional structure of their target region in the mRNA molecule. ${ }^{18}$ In this study, five antisense ODNs were initially selected with a GC content between $40 \%$ and $65 \%$ along the obtained partial sequence of rat CD40. Their blocking effects on CD40 expression were investigated in rat VSMCs. One of them (rAS3), located at position 46-63, proved to be a highly effective inhibitor of CD40 protein expression, attaining more than $80 \%$ inhibition at a concentration of $1 \mu \mathrm{M}$. In vitro, its blocking effect lasted for a maximum of three days post transfection, eventually due to degradation of the antisense ODNs and/or its dilution in the proliferating cells. Moreover, rAS3 specifically blocked CD154/CD40 interactions, as demonstrated by the lack of effect of the scrambled control ODN and decrease in VCAM-1 expression following CD154 but not TNF- $\alpha$ stimulation in rAS3 transfected rat VSMCs.

Collectively, these data confirm that rAS3 is not only a valuable tool that can be used to specifically interfere with 
CD154/CD50 interactions but is also valuable in investigating the pathological role of CD40 in chronic inflammatory diseases in vivo. Therefore, TNBS induced colitis in Wistar rats was employed as a model to evaluate the therapeutic potential of rAS3. This model features several characteristics of human Crohn's disease, including a Thl cell mediated pathogenic component ${ }^{19}$ which, however, may not be essential for initiation and/or perpetuation of the inflammatory response..$^{20} \mathrm{~A}$ role for CD154/CD40 interaction in this model has been revealed by increased expression of both CD154 and CD40 in the affected mucosa ${ }^{16}$ and the therapeutic effects achieved by administration of an anti-CD154 antibody. ${ }^{21}$

For successful administration of antisense ODNs in vivo they must reach a sufficient concentration in the affected tissue. The typical route of antisense ODN administration is by intravenous injection, which was used, for example, in most studies employing antisense ODNs against TNF- $\alpha$, ICAM-1, or VCAM-1 in IBD models. ${ }^{182}$ However, it may cause unexpected side effects and requires the use of extremely high dosages of the antisense ODN due to broad distribution of the injected nucleic acid. ${ }^{23}$ On the other hand, local administration may offer several advantages, including less side effects and a higher local concentration of the antisense ODN in the target tissue. In this study, rAS3 was first encapsulated in liposomes, which help the antisense ODN to cross the mucosal barrier, followed by administration through intracolonic injection.

A single application of the liposomal antisense ODN either prevented or greatly attenuated the severe inflammatory reaction following TNBS/ethanol enema, as judged by both macroscopic and microscopic evaluation. As revealed by immunohistochemistry, CD40 in the inflamed colon is mainly expressed by infiltrated monocytes and endothelial cells, which most likely represent the target of the antisense ODN in this study. Moreover, it is well known that CD154/ CD40 interactions between activated Thl cells and APCs/ endothelial cells in the affected mucosa greatly contribute to the secretion of proinflammatory cytokines, including IL-12, IFN $-\gamma$, and TNF- $\alpha$. $^{21}$ At the same time, expression of adhesion molecules on endothelial cells such as VCAM-1 is enhanced. This may result from exposure to proinflammatory cytokines and/or CD154/CD40 interactions directly.

RT-PCR analysis in fact demonstrated a significant reduction in VCAM-1 expression, which in turn may have contributed to the decrease in granulocyte infiltration revealed by histochemistry. Although neither T cell infiltration nor CD154 expression in the inflamed descending colon was affected, expression of IL-12 p40, which is thought to be mandatory for induction of a Thl immune response, was clearly blocked by rAS3 treatment. Moreover, recent data obtained with different knockout mice suggest that IL-12, but not IFN- $\gamma$, plays a pivotal role in TNBS induced colitis. ${ }^{25}$ This may also explain why we did not observe increased expression of IFN- $\gamma$ in our model. In either case, the aforementioned findings strongly suggest that rAS3 attenuates CD154/CD40 interactions in vivo, resulting in decreased mucosal inflammation in the subchronic IBD model employed.

Recently, the therapeutic effects of anti-CD154 antibodies have been verified in different chronic colitis models in mice. ${ }^{26}{ }^{27}$ Anti-CD154 antibody treatment in SCID mice has been shown to prevent the onset and progression of the disease. ${ }^{26}$ Even when administrated post colitis induction, anti-CD154 antibody treatment significantly improved the clinical and morphological appearance. ${ }^{26}{ }^{27}$ In contrast, antiCD154 antibody treatment has been shown to be ineffective in the mouse TNBS colitis model on establishment of the inflammatory response. ${ }^{21}$ The therapeutic effect of the CD40 antisense ODN in this study was demonstrated both by treatment prior to and post colitis induction. This suggests that perpetuation of the intestinal inflammation in the rat colitis model requires continuous or at least repeated CD154/ CD40 interactions.

In addition to the rat CD40 antisense ODNs, we have successfully explored several antisense ODNs against human CD40 in cultured endothelial cells, hence providing a platform for transferring this therapeutic approach to patients with chronic IBD. When compared with antiCD154 antibodies, CD40 antisense ODNs may provide the following advantages: no thromboembolic complications, ease of handling, higher stability, and low costs when administrated locally.

In summary, the results of this study suggest that CD40 antisense ODNs interfere with CD154/CD40 interactions with high potency and specificity both in vitro and in vivo. Therefore, they may provide an attractive therapeutic approach for the treatment of chronic inflammatory diseases, including Crohn's disease.

\section{ACKNOWLEDGEMENTS}

This study was supported by the Deutsche Forschungsgemeinschaft (SFB 402/C9 and GK 335). The expert technical assistance of Annette Bennemann is gratefully acknowledged.

\section{Authors' affiliations}

D Gao, A H Wagner, M Hecker, Department of Cardiovascular Physiology, University of Göttingen, Germany

S Schweyer, Department of Pathology, University of Göttingen, Germany

T Stojanovic, Department of Surgery, University of Göttingen, Germany S Fankhaenel, S Panzner, Novosom AG, Halle, Germany

Conflict of interest: None declared.

\section{REFERENCES}

1 van Kooten C, Bancheraeu J. CD40-CD40 ligand. J Leukoc Biol 2000;67:2-17

2 Schoenbeck U, Libby P. The CD40/CD154 receptor/ligand dyad. Cell Mol Life Sci 2001;58:4-43.

3 Castigli E, Alt FW, Davidson L, et al. CD40-deficient mice generated by recombinant-activating gene-2-deficient blastocyst complementation. Proc Natl Acad Sci U S A 1994;91:12135-9.

4 Renshaw BR, Fanslow WC III, Armitage RJ, et al. Humoral immune responses in CD40-ligand-deficient mice. J Exp Med 1994;180:1889-90.

5 Razanajaona D, Van Kooten C, Lebecque S, et al. Somatic mutations in human Ig variable genes correlate with the partially functional CD40-ligand in the X-linked hyper-lgM syndrome. J Immunol 1996;15:1492-8.

6 Hollenbaugh D, Mischel-Petty N, Edwards CP, et al. Expression of functional CD40 by vascular endothelial cells. J Exp Med 1995;182:33-40.

7 Karmann K, Hughes CC, Schechner J, et al. CD40 on human endothelial cells: inducibility by cytokines and functional regulation of adhesion molecule expression. Proc Natl Acad Sci U S A 1995;92:4342-6.

8 Xu J, Foy TM, Laman JD, et al. Mice deficient for the CD40 ligand. Immunity 1994; 1:423-31.

9 Neurath MF, Finotto S, Glimcher LH. The role of Th1/Th2 polarization in mucosal immunity. Nat Med 2002;8:567-73.

10 Battaglia E, Biancone L, Resegotti A, et al. Expression of CD40 and its ligand, CD40L, in intestinal lesions of Crohn's disease. Am J Gastroenterol 1999;94:3279-84.

11 Kawai T, Andrews D, Colvin RB, et al. Thromboembolic complications after treatment with monoclonal antibody against CD40 ligand. Nat Med 2000;6:114

12 Yamada A, Sayegh MH. The CD154-CD40 costimulatory pathway in transplantation. Transplantation 2002;73(suppl 1):S36-9.

13 Andre $\mathbf{P}$, Prasad KS, Denis CV, et al. CD40L stabilizes arterial thrombi by a beta3 integrin-dependent mechanism. Nat Med 2002;8:247-52.

14 Sakata K, Sakata A, Kong L, et al. Monocyte rescue of human T cells from apoptosis is CD40/CD154 dependent. Scand. J Immunol 1999;50:479-84.

15 Krzesz R, Wagner AH, Cattarruzza M, et al. Cytokine-inducible CD40 gene expression in vascular smooth muscle cells is mediated by nuclear factor $\kappa B$ and signal transducer and activator of transcription-1. FEBS Lett 1999; 453:191-6

16 Lienenlüke B, Stojanovic T, Fiebig T, et al. Thalidomide impairment of trinitrobenzene sulphonic acid-induced colitis in the rat-role of endothelial cell-leukocyte interaction. Br J Pharmacol 2001;133:1414-23.

17 Tone $M$, Tone $Y$, Fairchild PJ, et al. Regulation of CD40 function by its isoforms generated through alternative splicing. Proc Natl Acad Sci U S A 2001;98:1751-6. 
18 Myers KJ, Murthy S, Flanigan A, et al. Antisense oligonucleotide blockade of tumor necrosis factor- $\alpha$ in two murine models of colitis. J Phamacol Exp Ther 2003;304:41 1-24.

19 Singh B, Powire F, Mortensen NJ. Immune therapy in inflammatory bowel disease and models of colitis. Br J Surg 2001 :88:1558-69.

20 Hoffmann JC, Peters K, Henschke S, et al. Role of T lymphocytes in rat 2,4,6-trinitrobenzene sulphonic acid (TNBS) induced colitis: increased mortality after $\gamma \delta$ T cell depletion and no effect of $\alpha \beta$ T cell depletion. Gut 2001;48:489-95.

21 Stuber E, Strober W, Neurath M. Blocking the CD4OL-CD40 interaction in vivo specifically prevents the priming of T helper 1 cells through the inhibition of interleukin 12 secretion. J Exp Med 1996;183:693-8.

22 Rijcken E, Krieglstein CF, Anthoni C, et al. ICAM-1 and VCAM-1 antisense oligonucleotides attenuate in vivo leukocyte adherence and inflammation in rat inflammatory bowel disease. Gut 2002;51:529-35.
23 Galbraith WM, Hobson WC, Giclas PC, et al. Complement activation and hemodynamic changes following intravenous administration of phosphorothioate oligonucleotides in the monkey. Antisense Res Dev 1994;4:201-6.

24 Cong Y, Weaver CT, Lazenby A, et al. Colitis induced by enteric bacterial antigen-specific CD4+ T cells requires CD40-CD40 ligand interactions for a sustained increase in mucosal IL-12. J Immunol 2000;165:2173-82.

25 Tozawa K, Hanai H, Sugimoto K, et al. Evidence for the critical role of interleukin- 12 but not interferon- $\gamma$ in the pathogenesis of experimental colitis in mice. J Gastroenterol Hepatol 2003;18:578-87.

26 Liu Z, Geboes K, Colpaert S, et al. Prevention of experimental colitis in SCID mice reconstituted with CD45RB ${ }^{\text {high }} \mathrm{CD} 4^{+} \mathrm{T}$ cells by blocking the CD40-CD154 interactions. J Immunol 2000; 164:6005-14.

27 De Jong YP, Comiskey M, Kalled SL, et al. Chronic murine colitis is dependent on the CD154/CD40 pathway and can be attenuated by anti-CD154 administration. Gastroenterology 2000;119:715-23.

\section{EDITOR'S QUIZ: GI SNAPSHOT}

\section{An unusual cause of diarrhoea in a patient with colitis}

\section{Clinical presentation}

A 72 year old Asian woman, who grew up in Kenya and emigrated to the UK at the age of 36, was diagnosed with distal colitis on endoscopic and histological criteria. Treatment with mesalazine and oral corticosteroids led to remission.

Two months later she was readmitted with worsening diarrhoea, upper abdominal pain, and nausea. On physical examination she was afebrile and not tachycardic. There was abdominal tenderness. Blood tests revealed: white cell count $15.4 \times 10^{9} / \mathrm{l}$, C-reactive protein $30 \mathrm{mg} / \mathrm{l}$, and albumin $27 \mathrm{~g} / \mathrm{l}$. Stool specimens were negative for bacterial pathogens and Clostridium difficile. The working diagnosis was exacerbation of ulcerative colitis. She was treated with intravenous hydrocortisone (100 mg four times daily). Her symptoms worsened with severe abdominal pain and diarrhoea. The evaluation continued with upper gastrointestinal endoscopy. No macroscopic abnormality was seen. Haematoxylin-eosin staining of duodenal biopsies taken is shown in fig 1.

\section{Question}

What is the diagnosis and treatment of choice?

See page 96 for answer

This case is submitted by:

$$
\begin{array}{r}
\text { M Karmo, J Goh, R Boulton } \\
\text { Gastroenterology Unit, University Hospital Birmingham NHS trust, Queen } \\
\text { Elizabeth Hospital, Birmingham, UK } \\
\text { D S A Sanders } \\
\text { Department of Histopathology, South Warwickshire General Hospital } \\
\text { NHS Trust, UK }
\end{array}
$$

Correspondence to: Dr M Karmo, Birmingham Heartlands Hospital, 1 Sandy Way, Edgbaston, Birmingham B15 IEQ, UK; mkarmo@doctors.org.uk

doi: $10.1136 /$ gut.2004.043240
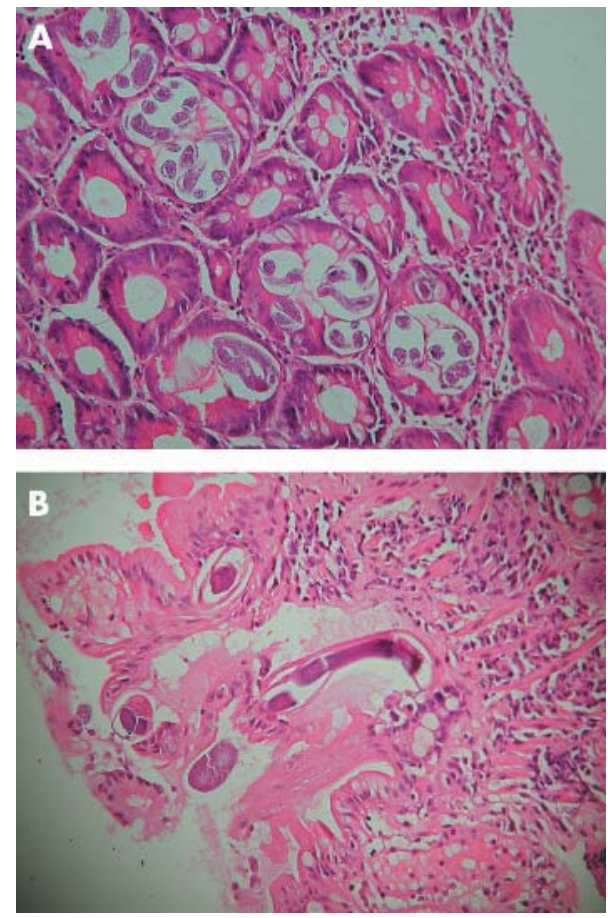

Figure 1 (A, B) Haematoxylin-eosin staining of duodenal biopsies. 